\title{
Using local redundancy to improve GNSS absolute positioning in harsh scenario
}

\author{
Antonio Angrisano ${ }^{1}$, Silvio Del Pizzo ${ }^{2}$, Salvatore Gaglione ${ }^{3}$, Salvatore Troisi ${ }^{3}$, Mario Vultaggio ${ }^{1}$ \\ ${ }^{1}$ G. Fortunato University, Benevento, Italy \\ ${ }^{2}$ iNav Geomatics Solutions, Massa Lubrense (NA), Italy \\ ${ }^{3}$ Parthenope University, Naples, Italy
}

\begin{abstract}
In GNSS context, absolute positioning is a widespread operational mode, largely used in many field activities as automotive, aerospace and ships navigation. The functional model of absolute positioning, relating pseudorange measurements to unknowns, is well defined, while its stochastic model, defining the behavior of measurement error, is currently under investigation and is the core of this work. A realistic model of measurement error can be used to define a weighting scheme, able to reduce the positioning errors; a weighted approach is mainly necessary in signal-degraded scenario, where pseudorange accuracies are significantly different and equally weighting all the measurements would yield very large errors.

The quality indicators, commonly adopted to define a pseudorange error model, are the signal-to-noise ratio and the satellite elevation angle. In this work, an additional indicator, the redundancy number, is introduced.

The redundancy numbers are the diagonal elements of the redundancy matrix and they represent the degree of controllability of the measurements; a large value of the redundancy number corresponds to a well-controlled measurement, while a small one corresponds to a leverage observation, with a high potential to influence the solution.

The benefits of using the proposed weighting schemes is demonstrated in urban canyon and with single (GPS only) or multiple (GPS/Glonass) constellations.
\end{abstract}

\section{Section: RESEARCH PAPER}

Keywords: GNSS; weighting schemes; redundancy matrix; local redundancy

Citation: Antonio Angrisano, Silvio Del Pizzo, Salvatore Gaglione, Salvatore Troisi, Mario Vultaggio, Using local redundancy to improve GNSS absolute positioning in harsh scenario, Acta IMEKO, vol. 7, no. 2, article 4, June 2018, identifier: IMEKO-ACTA-07 (2018)-02-04

Section Editor: Fabio Leccese, Università degli Studi di Roma Tre, Italy

Received January 15, 2018; In final form March 26, 2018; Published June 2018

Copyright: @ 2018 IMEKO. This is an open-access article distributed under the terms of the Creative Commons Attribution 3.0 License, which permits unrestricted use, distribution, and reproduction in any medium, provided the original author and source are credited

Corresponding author: Antonio Angrisano, e-mail: : a.angrisano@unifortunato.eu

\section{INTRODUCTION}

In GNSS context, scenarios characterized by bad signal quality and/or bad satellite geometry, usually due to natural or artificial obstacles, are considered harsh environments. Mountainous or highly urbanized areas are examples of harsh environments. In urban scenarios, multipath interference and non-line-of-sight (NLOS) phenomena typically affect the GNSS signals. The multipath interference happens when a signal is received through multiple paths; the combination of multiple signals causes a distortion of the correlation function (between received and locally generated signals) and yields a range error up to several tens of meters [1]. The NLOS reception happens when the direct signal is blocked and only reflected signals are received; this phenomenon is very common in urban scenario, yielding range errors of even $\mathrm{km}$ order [2]. The NLOS and multipath phenomena are even more widespread in high sensitivity receivers, which are able to receive very weak signals.

Absolute positioning, also called Single Point Positioning (SPP), is the most common GNSS operational mode. In fact, SPP mode is usually adopted by mass-market receivers, which are mounted on tablet, smartphones, car navigation devices, UAVs [3]-[5]; on these devices, SPP is often combined with other information sources, such as maps, inertial sensors or vision systems [6], [7], [8].

The aforementioned phenomena are responsible for the presence of blunders among the measurements, which can cause large position error. Two approaches are usually adopted to cope with this problem: 
- Receiver Autonomous Integrity Monitoring (RAIM) techniques, able to detect and reject blunders [9]-[11];

- Robust estimation techniques, able to absorb the effects of the blunders [12].

In this work, it has been followed a different approach, consisting in defining a suitable weighting scheme, able to deweighting potentially dangerous measurements. A capillary survey has been carried out on the existing measurement weighting schemes, which are tested in urban environment; afterwards, an additional quality criterion is proposed in order to limit the effect of potential blunders.

In literature, two quality indicators are typically associated to the GNSS measurements, in order to differently weighting them: satellite elevation angle and carrier-to-noise ratio.

Satellite elevation (ED) is considered a quality indicator of a measurement, because satellites at low elevation are usually noisier, due to typical behaviour of multipath, tropospheric and ionospheric errors [1].

The Signal-to-Noise ratio $(S / N)$, or better the Carrier-toNoise ratio $\left(C / N_{0}\right)$, is the ratio between the carrier power and the noise power per unit bandwidth and is usually expressed in Decibel-Hertz; $C / N_{0}$ is a measure of signal strength, so it is a suitable indicator of measurement quality [10].

The $E l$ and $C / N_{0}$ indicators are adoptable individually or synergistically to define a model of measurement error variance; a weighting scheme can be obtained, inverting the variances of the measurement errors.

The positioning solution is differently influenced by each measurement of a dataset; indeed, some measurements, called leverage observations, have high potential to influence the solution, while others are less influent. If a leverage observation is affected by a gross error, a very large solution error could occur; for this reason, the leverage observations are critical. It can be demonstrated that the concept of leverage observation is related to the local redundancy [13], which is well represented by the diagonal elements of redundancy matrix, called redundancy numbers.

In urban scenario, the satellite geometry is often weak and the presence of blunders is very common; in this conditions, a blunder on a leverage observation could strongly degrade the solution.

In this work, an innovative weighting scheme, which takes into account the local redundancy information, is proposed and its effectiveness is shown in a very harsh environment like an urban canyon. The proposed scheme can be superimposed to the existing weighting strategies, which involve the indicators El and $C / N_{0}$, and the benefits of this combined approach are demonstrated.

The proposed weighting scheme is applied to GPS only and to GPS/Glonass cases, demonstrating its usefulness in both configurations.

Below, the SPP operational mode is briefly described; afterwards, the existing weighting schemes, based on $E l$ and/or $C / N_{0}$, are described. Then the redundancy matrix, whose diagonal elements are the redundancy numbers, is defined and is linked up to the concept of local redundancy. The possible contribution of the redundancy matrix to a weighting strategy is shown. At the end, the proposed weightings are applied to real data and the results are discussed.

\section{SINGLE POINT POSITIONING}

In SPP, the functional model, defining the relationship between measurements and unknowns, is

$$
\boldsymbol{z}=H \Delta \boldsymbol{x}+\boldsymbol{\varepsilon}
$$

where $\boldsymbol{Z}$ is the vector of measurements, defined as the difference between measured and computed (with a priori information) pseudorange, $H$ is the design matrix, $\Delta \boldsymbol{x}$ is the state vector, containing the corrections to update the receiver coordinates and clock offset, $\varepsilon$ is the measurement error vector.

Equation (1), also referred to as measurement model, is a linearized equation; the receiver coordinates and clock offset are obtained correcting the a priori information with the estimated state vector.

The stochastic model, expressing the uncertainty of the measurement model, is:

$C=\left[\begin{array}{ccc}\sigma_{1}^{2} & \cdots & 0 \\ \vdots & \ddots & \vdots \\ 0 & \cdots & \sigma_{m}^{2}\end{array}\right]$

where $C$ is the variance-covariance matrix of the measurement errors; $\sigma_{i=1, \ldots, m}^{2}$ are the measurement error variances; $m$ is the number of measurements.

The matrix $C$ is diagonal, under the usual assumption of independent measurement errors.

The number of measurements is usually larger than the number of unknowns and the equation (1) is solved using weighted least squares (WLS) method.

WLS solution is:

$$
\widehat{\Delta \boldsymbol{x}}=\left(H^{T} W H\right)^{-1} H^{T} W \mathbf{z}
$$

where $W$ is the weighting matrix, usually set equal to the inverse of $C$.

\section{WEIGHTING SCHEMES REVIEW}

Most of weighting schemes in literature are based on $C / \mathrm{N}_{0}$, satellite elevation El or both; hence, the weighting strategies review is organized according to the considered quality indicators.

\subsection{Elevation-based weighting schemes}

The most widespread variance models for GPS carrier phase, based on satellite elevation, depend on $\sin (E l)$ or $\sin ^{2}(E l)$ functions [14], [15]; despite being designed for carrier phase, they were simply adapted for pseudorange observation [16], [17] as shown below:

$\sigma_{P R}^{2}=\frac{\sigma_{0}^{2}}{\sin (E l)}$

$\sigma_{P R}^{2}=\frac{\sigma_{0}^{2}}{\sin ^{2}(E l)}$

where $\sigma_{0}^{2}$ is the pseudorange error variance when $E l=90^{\circ}$.

Slight modifications to the previously mentioned models are proposed in literature [18], maintaining the concept that low elevation satellites correspond to noisy measurements:

$\sigma_{P R}^{2}=A^{2}+\frac{B^{2}}{\sin ^{2}(E l)}$

$\sigma_{P R}^{2}=\frac{\sigma_{0}^{2}}{\tan ^{2}(E l-\theta)}$

where $A, B, \theta$ are parameters to be defined according to the receiver and the operational scenario. 
A more complex variance model, which takes into account the contributions from several measurement error sources, is defined in [19], [20]; it is designed for Wide Area Augmentation System (WAAS) and it is shown below:

$\sigma_{P R}^{2}=\sigma_{U D R E}^{2}+F^{2}(E l) \cdot \sigma_{U I V E}^{2}+\sigma_{S N R}^{2}+\frac{\sigma_{m 45}^{2}}{\tan ^{2}(E l)}+\frac{\sigma_{t r v}^{2}}{\sin ^{2}(E l)}$

where $\sigma_{U D R E}=0.5 \mathrm{~m}$ is the variance of the supplied tropo-free and iono-free pseudorange correction, $\sigma_{U I V E}=0.5 \mathrm{~m}$ is the variance of the vertical ionosphere correction, $F$ is the obliquity factor, projecting vertical ionospheric variance into receiver satellite direction, $\sigma_{S N R}=0.22 \mathrm{~m}$ is the receiver noise variance, $\sigma_{m 45}=0.22 m$ is the variance of the multipath contribution at 45 degrees, $\sigma_{t r v}=0.15 \mathrm{~m}$ is the variance of the vertical tropospheric delay estimate.

The model (8) has been adapted to the case of GPS SPP in [21] as shown below:

$\sigma_{P R}^{2}=\sigma_{U R A}^{2}+\sigma_{\text {Iono }}^{2}+\sigma_{\text {Tropo }}^{2}+\sigma_{m p}^{2}$

where $\sigma_{U R A}^{2}$ is the User Range Accuracy (URA) related to the satellite ephemeris and clock, broadcast in the GPS navigation message, $\sigma_{\text {Iono }}^{2}$ is the accuracy related to ionosphere delay after Klobouchar model application, $\sigma_{\text {Tropo }}^{2}$ is the accuracy related to troposphere error after correction model application, $\sigma_{m p}^{2}$ is the accuracy related to multipath error.

\section{2. $\mathrm{C} / \mathrm{N}_{0}$-based weighting schemes}

In [14], a variance model for carrier phase observation is proposed; it is re-used and adapted in [2] for pseudorange measurement. The model is exclusively based on $\mathrm{C} / \mathrm{N}_{0}$ and has the following form:

$\sigma_{P R}^{2}=c \cdot 10^{-\frac{C / N_{0}}{10}}$

where $\mathrm{C}$ is a constant, empirically defined.

The model of Hartinger and Brunner [15] has been modified by Wieser and Brunner [22], introducing an additive term, and making the model parameters estimation in view of their application to carrier phase observable. Kuusniemi et al. [23], [24] adapt the Wieser and Brunner [22] model to pseudorange, tuning the parameters according to actual measurement errors and used equipment; the obtained variance model is:

$\sigma_{P R}^{2}=a+b \cdot 10^{-\frac{C / N_{0}}{10}}$

where $\mathrm{a}$ and $\mathrm{b}$ are the model parameters.

Kuusniemi [10] has estimated the parameters of model (11) for different scenarios (lightly and heavily degraded signal environment).

The Hartinger and Brunner [14] model has been the basis for Brunner et al. (1999) to build a carrier phase variance model, called sigma- $\Delta$, whose expression is very similar to (10), but with at the exponent a correction term proportional to the difference between the measured and the predicted (according to satellite elevation) $\mathrm{C} / \mathrm{N}_{0}$.

$\mathrm{Li}$ and $\mathrm{Wu}$ [26] have modified the model (11), adopting Euler's number as base of the exponentiation and the difference between the measured $\mathrm{C} / \mathrm{N}_{0}$ and its minimum threshold as exponent:

$\sigma_{P R}^{2}=a+b \cdot e^{k\left[\left(C / N_{0}\right)_{\text {min }}-C / N_{0}\right]}$

The parameters of the model are $\mathrm{a}, \mathrm{b}$ and $\mathrm{k}$ and are empirically determined.
Aminian [27] proposed an alternative expression for the pseudorange error variance without exponential form, as shown below:

$\sigma_{P R}^{2}=\sigma_{0}^{2} \frac{C / N_{0(\text { Zenith })}}{C / N_{0}}$

where $\sigma_{0}^{2}$ and $C / N_{0 \text { (Zenith) }}$ are respectively the theoretic variance and carrier-to-noise ratio in case of satellite at zenith and open-sky scenario.

\subsection{Weighting schemes based on both satellite elevation and $\mathrm{C} / \mathrm{N}_{0}$}

In literature, few weighting schemes, based on both satellite elevation and $\mathrm{C} / \mathrm{N}_{0}$, are available.

In [28] the following model is developed:

$\sigma_{P R}^{2}= \begin{cases}\frac{1}{\sin ^{2}(E l)} \Gamma, & \text { if } C / N_{0}<s_{1} \\ 1, & \text { if } C / N_{0} \geq s_{1}\end{cases}$

where

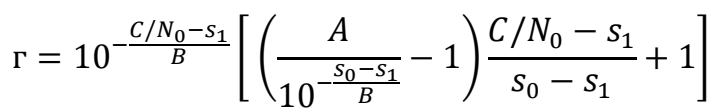

The term $s_{1}$ is a threshold, used as comparison for the measured $\mathrm{C} / \mathrm{N}_{0}$; if $C / N_{0} \geq s_{1}$, the measurement is considered accurate and the corresponding weight is set to 1 (the maximum possible value for a weight), otherwise a complex formula is adopted for the weight. The parameters in (14) and (15) are determined empirically as $S_{0}=10, A=30, B=30$.

In [29] a model, based on both satellite elevation and $C / \mathrm{N}_{0}$, designed for land navigation, is defined:

$\sigma_{P R}^{2}=k \cdot \frac{10^{-\frac{C / N_{0}}{10}}}{\sin ^{2}(E l)}$

The model (16) is clearly a fusion between the elevationbased model (5) and the $\mathrm{C} / \mathrm{N}_{0}$-based model (10). The constant $\mathrm{k}$ is 1 if the signal is received in line-of-sight (LOS) and 2 or $+\infty$ if the signal is received after reflection by obstacles surrounding the antenna (NLOS); in [29], a fish-eye camera is used to distinguish LOS and NLOS signals. In this work, a modified model (16) is used, where $\mathrm{k}$ is always equal to 1 .

\section{REDUNDANCY MATRIX}

The residuals $\boldsymbol{v}$, defined as the difference between the actual measurements and their estimated values $(\widehat{\mathbf{z}})$, are an indicator of the measurement mutual agreement [13]:

$\boldsymbol{v}=\boldsymbol{z}-\hat{\boldsymbol{z}}=\boldsymbol{z}-H \widehat{\Delta \boldsymbol{x}}$

It can be simply demonstrated that the relationship between the measurement errors $\boldsymbol{\varepsilon}$ and the LS residuals, considering equally weighted measurements, is:

$\boldsymbol{v}=R \boldsymbol{\varepsilon}$

The matrix $R$ is called "Redundancy Matrix" and has the following expression:

$R=I-H\left(H^{T} H\right)^{-1} H^{T}$

The matrix $R$ has $m$ rows and $m$ columns, its trace is equal to the total redundancy (or the degree of freedom) of the equation, that is $(m-n)$. The $\mathrm{i}$-th diagonal element of $R, r_{i}$ with $i=1, \ldots m$, is called "redundancy number" and is the contribution of the $\mathrm{i}$-th measurement to the total redundancy 
[31]. The redundancy number assumes values between 0 and 1. Small values of $r_{i}$ (near 0 ) correspond to measurements providing little contribution to total redundancy and so hardly controlled; on the other hand, approximately equal values of $r_{i}$ are desirable, meaning that every measurement is controllable.

Measurements with small $r_{i}$ values are leverage observations and have high potential to influence the solution; if a blunder or a large bias is present on a leverage observation, harmful effects can be evident on the positioning. In this context, de-weighting leverage observations could be a successful strategy, above all in environments, as urban canyon, characterized by frequent gross errors.

In Table 1, an example of GPS geometry, in terms of satellite elevations $(E l)$ and azimuths $(A z)$, is shown; from $E l$ and $A z$, the design matrix can be computed and, consequently, the redundancy matrix $R$ is obtained. The diagonal elements of $R$ are the redundancy numbers.

In Figure 1, the sky-plot of the satellite geometry, detailed in Table 1, is shown; all the listed satellites are considered, so the number of visible GPS satellites is 10 and the PDOP (Position Dilution of Position) value is 1.59 . The total redundancy of the measurement model is $6(=m-n)$, therefore the average redundancy number is 0.6. The sky-plot is accompanied by the values of the redundancy numbers and it is evident that all the values are near the average.

In Figure 2, the sky-plot of the satellite geometry, detailed in Table 1, is shown, but satellites with PRNs 17, 19 and 25 are excluded; consequently, the number of visible GPS satellites is 7 and the PDOP value raises to 2.15. The total redundancy is 3 , so the average redundancy number is about 0.43 . The redundancy numbers are all around or above the average value, except the one corresponding to satellite 22 , which is very low and so can be considered a leverage observation. From Figure 2 , it is evident a geometric interpretation of leverage

Table 1. Example of GPS geometry, in terms of satellite elevation and azimuth.

\begin{tabular}{ccc}
\hline PRN & El deg & Az deg \\
\hline 2 & 12 & 30 \\
3 & 24 & 50 \\
5 & 45 & 80 \\
6 & 21 & 119 \\
9 & 65 & 130 \\
11 & 30 & 170 \\
17 & 81 & 190 \\
19 & 20 & 250 \\
22 & 19 & 282 \\
25 & 30 & 348 \\
\hline
\end{tabular}

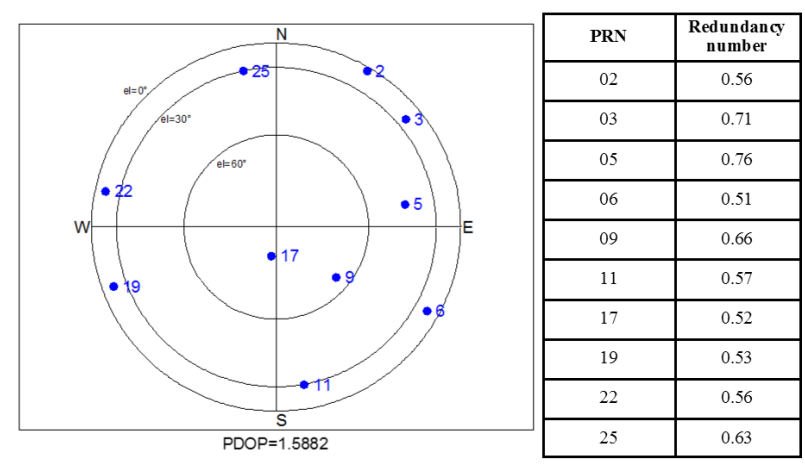

Figure 1. Sky-plot of GPS satellite configuration; the total redundancy is 6 .

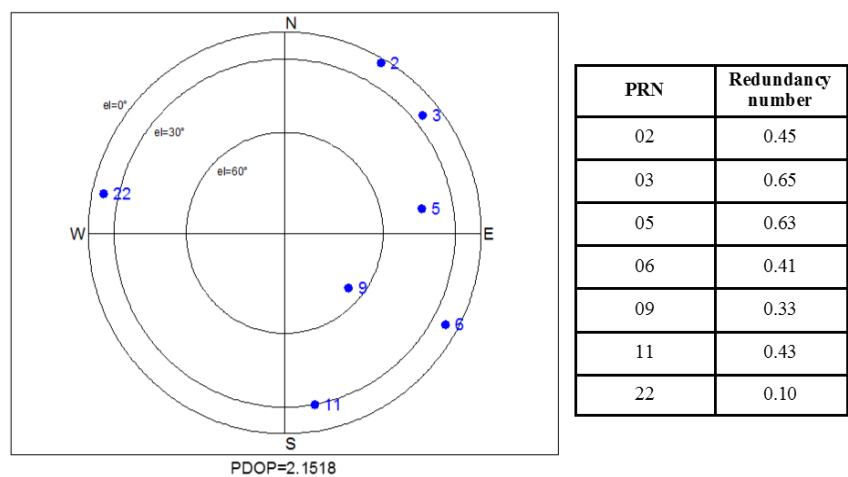

Figure 2. Sky-plot of GPS satellite configuration; the total redundancy is 3.

observation, for GPS SPP, as a measurement corresponding to a satellite "isolated" with respect to the others.

In case of multi-constellation approach, a redundancy number is null when a single satellite from a system is present. For instance, in a GPS/Glonass combination, if there is only one GPS measurement and several Glonass, the redundancy number corresponding to the unique GPS observation is 0 and the observation is uncontrollable (because it is the only one able to estimate the clock offset to GPS time). The same concept is valid for any GNSS combination, including the European GNSS, Galileo [30], or the Chinese one BeiDou.

\section{CONTRIBUTION OF REDUNDANCY MATRIX TO WEIGHTING SCHEMES}

The diagonal elements of the redundancy matrix, the redundancy numbers $r_{i}$, represent the local reliability of the measurement model and so the controllability of the measurements [31].

Measurements with low $r_{i}$ are critical for the solution, because they can strongly influence it. For this reason, an effective weighting strategy should take into account the redundancy number, above all in scenarios with high probability of blunders.

In [32] the redundancy number is used, together with several other parameters, as an indicator of the measurement quality for robust estimation. In [10] the extra-diagonal elements of the redundancy matrix are used to evaluate the correlation among the measurements, in order to avoid erroneous blunder rejections. Recently, Falco et al. [33] adopted a purely geometric strategy to identify measurements which could strongly influence the solution, in order to de-weight them.

In this paper, the information contained in the redundancy matrix is used to correct the original weighting matrix, which could be defined according to one of the previously described methods. In general, the weight $w_{i}$, associated to the i-th measurement, is obtained inverting the pseudorange error variance $\sigma_{P R i}^{2}$.

The contribution of a redundancy number to the weighting strategy could be introduced making $w_{i}$ proportional to $r_{i}$ as shown below:

$$
w_{i}= \begin{cases}\frac{r_{i}}{\sigma_{P R i}^{2}} & , \text { in case of redundant measurements } \\ \frac{1}{\sigma_{P R i}^{2}} & , \text { in case of lack of redundancy }\end{cases}
$$

The redundancy numbers are 0 in case of lack of redundancy and the scheme (20) takes into account this possibility. 
The strategy is tested on a multi-constellation static data collection, carried out in urban scenario, and demonstrated its efficiency in both GPS only and GPS/Glonass cases, exhibiting significant improvements on positioning accuracy.

The same strategy could be simply extended to GNSS velocity estimation [34].

\section{TEST}

To demonstrate the benefits of including the redundancy number into a weighting scheme in difficult environment, a static data collection is carried out in urban scenario; specifically, the test location is the "Centro Direzionale di Napoli" (CDN), a district of Naples (Italy) characterized by several skyscrapers. A specific point in CDN is surveyed with topographical methods, in order to determine its coordinates with $\mathrm{mm}$ order. The used equipment consists in a NVS receiver, a single frequency, double constellation (GPS/GLONASS) and high-sensitivity device, connected to a patch antenna; the device is placed on the defined point to collect pseudorange measurements for some hours; the collected data are processed in single point to test several weighting schemes.

In Figure 3, the considered point (indicated as P) is shown and the urban canyon scenario is evident.

The data collection was carried out on $1^{\text {st }}$ July 2016; the NVS receiver was placed on $\mathrm{P}$ point for about 2 hours. In Figure 4, the number of GPS/Glonass visible satellites and the corresponding PDOP values during the session are shown.

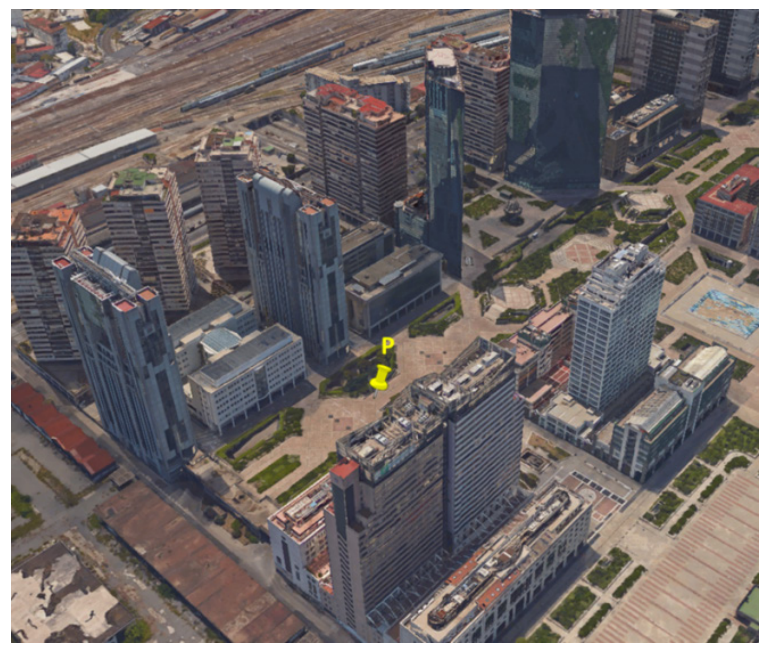

Figure 3. Test location.

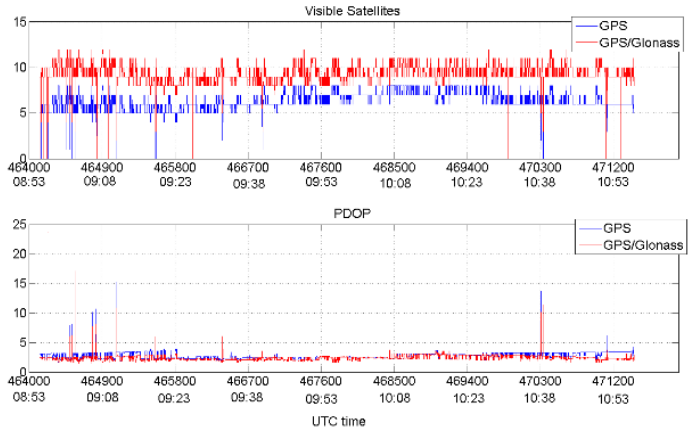

Figure 4. Number of visible GPS/Glonass satellites and corresponding PDOP during session.
The number of GPS visible satellites ranges between 0 and 8 , with an average of 6.4. The maximum number of Glonass visible satellites is 5 , while the maximum number of combined GPS/Glonass satellites is 12 .

The GPS solution availability, defined as the time percentage where the solution can be computed (at least 4 satellites should be visible), is about $99.3 \%$, while the GPS/Glonass solution availability is about $99.5 \%$.

Despite the urban canyon scenario, the solution availability is high, owing to the use of a high-sensitivity receiver, able to acquire very weak signals. The rapid oscillations of the number of visible satellites is due to frequent signal loss, typical of hostile environment.

In GPS only case, the PDOP average value is about 2.7, with maximum and minimum respectively about 15 and 1.6; in GPS/Glonass case, minimum, maximum and mean values are respectively 1.5, 17 and 2.4. The maximum PDOP value in GPS/Glonass case happens when GPS satellites are less than four, so in that time window GPS DOP cannot be computed.

\section{RESULTS}

Several weighting strategies are described in section 2; among them, some techniques are chosen to process data collected in urban scenario, in order to compare their performance.

The considered configurations, implementing GNSS SPP with a distinct weighting scheme, are:

- The one using weights defined by formula (5), shortly indicated as ELV1;

- The one using weights defined by formula (9), shortly indicated as ELV2;

- The one using weights defined by formula (11), shortly indicated as CN01;

- The one using weights defined by formula (13), shortly indicated as $\mathrm{CN} 02$;

- The one using weights defined by formula (14), shortly indicated as ELVCN01;

- The one using weights defined by formula (16), shortly indicated as ELVCN02.

The previously mentioned configurations are compared with the baseline one, where all the measurements are equally weighted, shortly indicated as EQW.

The figure of merits adopted for the comparison are the mean, RMS and maximum errors of both horizontal and vertical components of position.

In Table 2 the configuration performance, in case of GPS only, are summarized.

Table 2. Figure of merits obtained with GPS only, using classic weighting methods.

\begin{tabular}{lcccccc}
\hline & \multicolumn{2}{c}{ Mean m } & \multicolumn{2}{c}{ RMS m } & \multicolumn{2}{c}{ Maximum m } \\
& Hor. & Vert. & Hor. & Vert. & Hor. & Vert. \\
\hline EQW & 16,0 & 18,2 & 44,9 & 45,9 & 351,6 & 316,6 \\
ELV1 & 10,4 & 12,9 & 28,2 & 33,3 & 187,7 & 224,0 \\
ELV2 & 13,0 & 12,2 & 38,6 & 29,0 & 283,3 & 218,0 \\
CN01 & 15,2 & 17,2 & 43,1 & 44,3 & 340,1 & 300,9 \\
CN02 & 15,4 & 17,6 & 43,4 & 45,1 & 341,0 & 305,8 \\
ELVCN01 & 8,4 & 9,9 & 22,4 & 27,9 & 158,5 & 194,9 \\
ELVCN02 & 5,9 & 5,8 & 14,9 & 19,5 & 130,1 & 173,4 \\
\hline
\end{tabular}


The results obtained with the EQW configuration is clearly inaccurate; in fact, the errors, on both components and for each figure of merit, are very large owing to the frequent blunders in the dataset. The considered weighting schemes reduce drastically the errors; the models based uniquely on satellite elevation (ELV1 and ELV2) show better performance with respect to models based uniquely on carrier-to-noise ratio (CN01 and CN02). The methods which consider both quality indicators (ELVCN01 and ELVCN02) demonstrate significant improvements relatively to the other configurations. In particular, the model introduced by Tay and Marais [29], i.e. ELVCN02 configuration, shows the best performance for the considered data session; indeed RMS and mean errors are reduced, with respect to EQW configuration, between $58 \%$ and $68 \%$, and the maximum horizontal and vertical errors are reduced respectively by $63 \%$ and $45 \%$.

The considered weighting schemes are augmented, using information embedded in the redundancy matrix as described in previous sections; the configurations using this information are shortly indicated as RDM. For instance, a configuration using the elevation-based model (5), augmented with redundancy matrix-based approach, is shortly indicated as ELV1+RDM. In Table 3, the performance of the configurations, obtained combining the considered classical weighting schemes with redundancy matrix information, are resumed. Moreover, in the table, the percentage improvements, with respect to corresponding configurations without redundancy matrix use, are reported alongside the errors. It is evident that the introduction into the weighting schemes of information from redundancy matrix brings to significant improvements. The best configuration is ELVCN02+RDM, characterized by horizontal mean, RMS and maximum errors respectively about 4.5, 8.8 and 87.2 meters, a very good performance considering the strongly degraded scenario.

The same analysis, carried out for GPS only, is provided for GPS/Glonass multi-constellation case. In Table 4 the GPS/Glonass configuration performance are summarized. The addition of Glonass measurements to GPS ones does not improve all the considered figure of merits, as Glonass measurements are often very noisy; in detail, GPS/Glonass configurations have better performance in horizontal plane, but worse performance in vertical direction. The errors of EQW configuration are very large, because there are several blunders among the measurements, from both used systems; the Glonass use allows a reduction in maximum horizontal of about 130

Table 3. Figure of merits obtained with GPS only, using classic weighting methods, augmented with information from redundancy matrix.

\begin{tabular}{lcccccc}
\hline & \multicolumn{3}{c}{ Mean m } & \multicolumn{2}{c}{ RMS m } & \multicolumn{2}{c}{ Maximum m } \\
& Horiz. & Vert. & Horiz. & Vert. & Horiz. & Vert. \\
\hline \multirow{2}{*}{ EQW+RDM } & 14,0 & 15,5 & 36,4 & 37,2 & 276,5 & 241,5 \\
& $(12,2 \%)$ & $(14,9 \%)$ & $(19,0 \%)$ & $(18,9 \%)$ & $(21,4 \%)$ & $(23,7 \%)$ \\
ELV1+RDM & 8,0 & 9,0 & 18,1 & 22,8 & 121,1 & 154,7 \\
& $(22,9 \%)$ & $(29,8 \%)$ & $(35,7 \%)$ & $(31,6 \%)$ & $(35,5 \%)$ & $(30,9 \%)$ \\
ELV2+RDM & 10,6 & 9,1 & 27,8 & 20,9 & 186,2 & 136,5 \\
& $(18,4 \%)$ & $(25,3 \%)$ & $(27,8 \%)$ & $(27,9 \%)$ & $(34,3 \%)$ & $(37,4 \%)$ \\
CN01+RDM & 13,0 & 14,2 & 33,7 & 34,7 & 261,5 & 228,1 \\
& $(14,4 \%)$ & $(17,6 \%)$ & $(21,7 \%)$ & $(21,6 \%)$ & $(23,1 \%)$ & $(24,2 \%)$ \\
CN02+RDM & 13,3 & 14,7 & 34,4 & 35,8 & 261,7 & 230,3 \\
& $(13,7 \%)$ & $(16,6 \%)$ & $(20,9 \%)$ & $(20,6 \%)$ & $(23,2 \%)$ & $(24,7 \%)$ \\
ELVCN01+RDM & 6,3 & 6,3 & 13,6 & 17,6 & 108,1 & 125,0 \\
& $(24,7 \%)$ & $(35,7 \%)$ & $(39,4 \%)$ & $(36,9 \%)$ & $(31,8 \%)$ & $(35,9 \%)$ \\
ELVCN02+RDM & 4,5 & 3,3 & 8,8 & 11,4 & $87,2($ & 115,9 \\
& $(23,6 \%)$ & $(43,8 \%)$ & $(41,1 \%)$ & $(41,7 \%)$ & $33,0 \%)$ & $(33,2 \%)$ \\
\hline
\end{tabular}

Table 4. Figure of merits obtained with GPS/Glonass, using classic weighting methods.

\begin{tabular}{lcccccc}
\hline & \multicolumn{2}{c}{ Mean m } & \multicolumn{2}{c}{ RMS m } & \multicolumn{2}{c}{ Maximum m } \\
& Horiz. & Vert. & Horiz. & Vert. & Horiz. & Vert. \\
\hline EQW & 14,6 & 28,0 & 33,5 & 62,3 & 212,9 & 343,2 \\
ELV1 & 8,7 & 19,0 & 17,9 & 40,4 & 112,0 & 205,7 \\
ELV2 & 12,7 & 12,7 & 37,4 & 29,7 & 268,9 & 217,7 \\
CN01 & 13,7 & 26,2 & 31,8 & 59,2 & 196,5 & 335,1 \\
CN02 & 14,0 & 26,9 & 32,3 & 60,5 & 202,0 & 339,0 \\
ELVCN01 & 7,1 & 14,4 & 14,4 & 32,0 & 97,5 & 178,9 \\
ELVCN02 & 5,3 & 8,4 & 10,4 & 21,2 & 92,4 & 162,0 \\
\hline
\end{tabular}

meters. As for GPS only case, the weighting schemes based uniquely on satellite elevation (ELV1 and ELV2) demonstrate better performance than models based uniquely on carrier-tonoise ratio (CN01 and CN02); models exploiting both quality indicators show the best results.

In Table 5, the figure of merits of the GPS/Glonass configurations, using classical weighting schemes augmented with redundancy matrix information, are resumed; in the table, the percentage improvements, with respect to corresponding configurations without redundancy matrix use, are reported alongside the errors. The benefits of using redundancy matrix in weighting schemes are evident even in GPS/Glonass case; indeed, all the considered errors are strongly reduced. The best configuration is ELVCN02+RDM, characterized by horizontal mean, RMS and maximum errors respectively about 4.5, 8.1 and 73.6 meters, a very good performance considering the strongly degraded scenario and slightly lower with respect to GPS best configuration.

The use of information derived from redundancy matrix demonstrates very good performance for both GPS only and GPS/Glonass cases. The error reductions are impressive for each considered configuration and figure of merit. In particular, the best configuration is ELVCNO2+RDM, using $C / N_{0}$, satellite elevation and local redundancy as parameters for weighting; the obtained performances are characterized by mean and RMS horizontal errors respectively below 5 and 10 meters, and maximum horizontal error about 80 meters.

The considered approach, taking into account the information contained into the redundancy matrix, demonstrates its effectiveness, owing to the ability to deweighting the geometrically dangerous measurements.

Table 5. Figure of merits obtained with GPS/Glonass, using classic weighting methods, augmented with information from redundancy matrix.

\begin{tabular}{lcccccc}
\hline & \multicolumn{2}{c}{ Mean m } & \multicolumn{2}{c}{ RMS m } & \multicolumn{2}{c}{ Maximum m } \\
& Horiz. & Vert. & Horiz. & Vert. & Horiz. & Vert. \\
\hline \multirow{2}{*}{ EQW+RDM } & 13,5 & 19,8 & 32,9 & 44,1 & 236,6 & 266,0 \\
& $(7,5 \%)$ & $(29,4 \%)$ & $(1,9 \%)$ & $(29,1 \%)$ & $(11,1 \%)$ & $(22,5 \%)$ \\
ELV1+RDM & 7,7 & 12,1 & 15,5 & 25,9 & 94,8 & 153,1 \\
& $(12,3 \%)$ & $(36,0 \%)$ & $(13,2 \%)$ & $(36,0 \%)$ & $(15,3 \%)$ & $(25,5 \%)$ \\
ELV2+RDM & 11,0 & 10,0 & 29,5 & 23,0 & 212,0 & 164,8 \\
& $(13,5 \%)$ & $(21,1 \%)$ & $(21,0 \%)$ & $(22,5 \%)$ & $(21,2 \%)$ & $(24,3 \%)$ \\
CN01+RDM & 12,5 & 18,0 & 30,6 & 41,1 & 224,7 & 242,2 \\
& $(8,8 \%)$ & $(31,3 \%)$ & $(3,9 \%)$ & $(30,7 \%)$ & $(14,4 \%)$ & $(27,7 \%)$ \\
CN02+RDM & 12,8 & 18,6 & 31,2 & 42,3 & 224,3 & 249,4 \\
& $(8,5 \%)$ & $(30,7 \%)$ & $(3,4 \%)$ & $(30,1 \%)$ & $(11,1 \%)$ & $(26,4 \%)$ \\
ELVCN01+RDM & 6,1 & 8,6 & 11,9 & 19,6 & 86,1 & 122,6 \\
& $(14,1 \%)$ & $(40,0 \%)$ & $(17,6 \%)$ & $(38,9 \%)$ & $(11,7 \%)$ & $(31,5 \%)$ \\
ELVCN02+RDM & 4,5 & 4,6 & 8,1 & 12,3 & 73,6 & 109,1 \\
& $(14,8 \%)$ & $(45,4 \%)$ & $(22,8 \%)$ & $(41,7 \%)$ & $(20,4 \%)$ & $(32,7 \%)$ \\
\hline
\end{tabular}




\section{CONCLUSIONS}

In GNSS context, some environments, such as urban canyons, are critical, owing to the likely presence of blunders among the measurements, which yields very large position errors. In these conditions, it is essential to adopt a suitable method to differently weight the measurements, in order to limit the blunder effects. In literature, several schemes exist, based on two quality indicators: satellite elevation and carrierto-noise ratio.

In this paper, the most representative weighting schemes for GNSS absolute positioning are tested in urban scenario and their performance are compared. The best results are obtained with the weighting strategies based on both the abovementioned quality indicators.

The concept of local redundancy refers to the degree of controllability of the observations; for instance, a measurement with low local redundancy is difficultly controlled and, if affected by gross errors, it strongly influences the solution. The redundancy matrix relates the measurement errors to the residuals and its diagonal elements are the redundancy numbers, representing the local redundancy. In this work, a strategy to include the local redundancy into the existing weighting schemes is suggested. In the proposed approach, each weight value is set proportionally to the corresponding local redundancy number. In this way, the most influencing measurements (i.e. the leverage measurements) are de-weighted and their effects on the solution are limited.

The considered weighting schemes are applied to process in SPP a 2-hour dataset, collected in urban scenario; both GPS only and multi-constellation GPS/Glonass configurations are analysed. The obtained results demonstrate the effectiveness of the proposed weighting strategy; indeed, the position errors, using the weighting scheme based on redundancy numbers, are significantly reduced for all the considered configurations.

The best performances are attained with a weighting scheme, comprising the two classical quality indicators, satellite elevation and carrier-to-noise ratio, and the redundancy number; in single point positioning, a horizontal accuracy below 10 meters is obtained in a severe urban scenario.

\section{REFERENCES}

[1] Kaplan, E.D. and Hegarty, J. (2006). Understanding GPS: Principles and Applications. Artech House Mobile Communications Series.

[2] Groves, P. and Jiang, Z. (2013). Height aiding cn0 weighting and consistency checking for gnss nlos and multipath mitigation in urban areas. Journal of Navigation, 66(5), 653-669.

[3] Van Sickle, J. (2015). GPS For Land Surveyors, CRC Press, New York, NY, USA.

[4] Yoon, D., Kee, C., Seo, J. and Park, B. (2016). Position Accuracy Improvement by Implementing the DGNSS-CP Algorithm in Smartphones. Sensors, 16(6), 16 pages.

[5] Bodini, I., Lancini, M., Pasinetti, S., \& Vetturi, D. (2014), Techniques for on-board vibrational passenger comfort monitoring in public transport. ACTA IMEKO, 3(4), 32-37.

[6] Petritoli, E., Giagnacovo, T. and Leccese, F. (2014). Lightweight GNSS/IRS integrated navigation system for UAV vehicles. Proceedings of 2014 IEEE International Workshop on Metrology for Aerospace, MetroAeroSpace 2014.

[7] Fontanelli, D., Macii, D., \& Rizano, T. (2015). A fast and lowcost vision-based line tracking measurement system for robotic vehicles. ACT A IMEKO, 4(2), 90-99.

[8] Ackermann, S., Angrisano, A., Del Pizzo, S., Gaglione, S., Gioia, C., Troisi, S. (2014). Digital surface models for GNSS mission planning in critical environments. Journal of Surveying Engineering, 140 (2), art. no. 0000119. DOI: 10.1061/(ASCE)SU.1943-5428.0000119

[9] Brown, R. G. and Chin, G. Y. (1997). GPS RAIM: calculation of threshold and protection radius using chi-square methods-a geometric approach. Global Positioning System: Institute of Navigation, 5, 155-179.

[10] Kuusniemi, H. (2005). User-level reliability and quality monitoring in satellite based personal navigation. Ph.D. dissertation, Tampere University of Technology, Tampere, Finland.

[11] Castaldo, G., Angrisano, A., Gaglione, S. and Troisi, S. (2014). PRANSAC: An Integrity Monitoring Approach for GNSS Signal Degraded Scenario. International Journal of Navigation and Observation, Volume 2014, Article ID 173818, 11 pages.

[12] Knight, N. and Wang, J. (2009). A Comparison of Outlier Detection Procedures and Robust Estimation Methods in GPS Positioning. Journal of Navigation, 62(4), pp. 699-709.

[13] Leick, A. (2004). GPS Satellite Surveying. JohnWiley and Sons, Inc.

[14] Hartinger, H. and Brunner, F. (1999). Variances of GPS phase observations: The sigma- $\varepsilon$ model. GPS Solutions, 2, 35-43.

[15] Collins, J.P. and Langley, R.B. (1999). Possible Weighting Schemes for GPS Carrier Phase Observations in the Presence of Multipath. Final contract report for the U.S. Army Corps of Engineers Topographic Engineering Center, No. DAAH04-96C-0086 / TCN 98151, March, 33 pp.

[16] Petovello, M. (2003). Real-time Integration of a Tactical-Grade IMU and GPS for High-Accuracy Positioning and Navigation. $\mathrm{PhD}$ Thesis, Department of Geomatics Engineering, University of Calgary, Canada.

[17] Wieser, A. (2007). How important is GNSS observation weighting? GNSS Solutions Column, Inside GNSS, JanuaryFebruary Issue, pp. 26-28, 2007.

[18] Rahemi, N., Mosavi, M. R., Abedi, A. A. and Mirzakuchaki, S. (2014). Accurate Solution of Navigation Equations in GPS Receivers for Very High Velocities Using Pseudorange Measurements. Advances in Aerospace Engineering, Volume 2014, Article ID 435891, 8 pages.

[19] RTCA (2006). Minimum Operational Performance Standards for Global Positioning System/Wide Area Augmentation System Airborne Equipment, DO-229D.

[20] Walter, T. and Enge, P. (1995). Weighted RAIM for precision approach. Proceedings of the 8th International Technical Meeting of the Satellite Division of The Institute of Navigation (ION GPS 1995), Palm Springs, CA, US.

[21] Angrisano, A., Gaglione, S. and Gioia, C. (2012). RAIM algorithms for aided GNSS in Urban scenario. Proceedings of the Ubiquitous Positioning, Indoor Navigation, and Location Based Service (UPINLBS '12), Helsinki, Finland.

[22] Wieser, A. and Brunner, F.K. (2000). An extended weight model for GPS phase observations. Earth Planets Space, 52, pp $777-$ 782.

[23] Kuusniemi, H., Lachapelle, G. and Takala J. H. (2004). Position and velocity reliability testing in degraded GPS signal environment. GPS Solutions, 8(4), pp 226-237.

[24] Kuusniemi, H., Wieser, A., Lachapelle, G. and Takala, J. (2007). User-level reliability monitoring in urban personal satellite navigation. IEEE Transactions on Aerospace and Electronic Systems, 43(4).

[25] Brunner, F., Hartinger, H. and Troyer, L. (1999). GPS signal diffraction modelling: the stochastic sigma- $\Delta$ model. Journal of Geodesy, 73, 259-267.

[26] Li, J. and Wu, M. (2009). The improvement of positioning accuracy with weighted least square based on snr. Proceedings of the 5th International Conference on Wireless Communications, Networking and Mobile Computing, Beijing, China.

[27] Aminian, B. (2011). Investigation of GPS observations for indoor GPS/INS integration. MSc thesis, Department of Geomatics Engineering, University of Calgary, Canada. 
[28] Realini, E. and Reguzzoni, M. (2013). GoGPS: Open source software for enhancing the accuracy of low-cost receivers by single-frequency relative kinematic positioning. Measurement Science and Technology, 24(11).

[29] Tay, S. and Marais, J. (2013). Weighting models for GPS Pseudorange observations for land transportation in urban canyons. Proceedings of the 6th European Workshop on GNSS Signals and Signal Processing, Munich, Germany.

[30] Angrisano, A., Gaglione, S., Gioia, C., Borio, D., FortunyGuasch, J. (2013). Testing the test satellites: The Galileo IOV measurement accuracy. 2013 International Conference on Localization and GNSS, ICL-GNSS 2013, art. no. 6577253. DOI: 10.1109/ICL-GNSS.2013.6577253

[31] Schaffrin, B. (1997). Reliability measures for correlated observations. Journal of Surveying Engineering, 123(3), 126-137.
[32] Wieser, A. (2001). A Fuzzy System for Robust Estimation and Quality Assessment of GPS Data for Real-Time Applications. Proceedings of the 14th International Technical Meeting of the Satellite Division of The Institute of Navigation (ION GPS 2001), Salt Lake City, UT, US.

[33] Falco, G., Nicola, M. and Falletti, E. (2016). Constellation-Aware Method for Computing the Covariance Matrix of GNSS Measurements. Proceedings of the European Navigation Conference 2016, Helsinki, Finland.

[34] Freda, P., Angrisano, A., Gaglione, S., Troisi, S. (2015). Timedifferenced carrier phases technique for precise GNSS velocity estimation. GPS Solutions, 19 (2), pp. 335-341. DOI: 10.1007/s10291-014-0425-1. 